\title{
Nonlocal pseudopotentials and magnetic fields
}

\author{
Chris J. Pickard \\ TCM Group, Cavendish Laboratory, Madingley Road, \\ Cambridge, CB3 OHE, United Kingdom \\ Francesco Mauri \\ Laboratoire de Minéralogie-Cristallographie de Paris, \\ Université Pierre et Marie Curie, 4 Place Jussieu, 75252, Paris, Cedex 05, France
}

(Dated: November 12, 2018)

\begin{abstract}
We show how to describe the coupling of electrons to non-uniform magnetic fields in the framework of the widely used norm-conserving pseudopotential approximation for electronic structure calculations. Our derivation applies to magnetic fields that are smooth on the scale of the core region. The method is validated by application to the calculation of the magnetic susceptibility of molecules. Our results are compared with high quality all electron quantum chemical results, and another recently proposed formalism.
\end{abstract}

PACS numbers: 71.15.-m, 71.45.Gm, 71.15.Dx, 71.15.Mb

The coupling of the electrons in matter with probing electromagnetic fields or charged particles provides the basis for nearly all known analytical experimental techniques. However, the most computationally efficient schemes for the first principles prediction (and hence interpretation) of the experimental observables require the use of approximate, and crucially, nonlocal Hamiltonians. Most notably, the use of nonlocal pseudopotentials, along with with density functional theory, is often referred to as the Standard Model of modern electronic structure theory. This is not without justification. The computational efficiency, and accuracy of modern first principles pseudopotentials has allowed a vast range of problems to be solved for realistic materials. However, whatever the successes of this method, it is still unclear how nonlocal Hamiltonians should be coupled to arbitrary magnetic fields. In the specific case of uniform magnetic fields described with the symmetric-gauge vector-potential, we have derived the correct Hamiltonian by developing what we called the gauge including projector augmented wave (GIPAW) method [1]. In this Letter we derive the pseudopotential Hamiltonian that describes the coupling between the electrons and a smooth non-uniform magnetic field represented by an arbitrary vector-potential gauge.

We are not alone in this quest. Recently Ismail-Beigi, Chang and Louie 2], to whom we refer the reader for a more complete summary of earlier work in this field, proposed a scheme (hereafter referred to as the ICL method) which sought to finally provide a rigorous derivation of a closed form for the coupling of nonlocal systems to arbitrary electromagnetic fields. While the widespread use of nonlocal pseudopotentials clearly provided the motivation and applications for their method, they attempted to tackle the problem of a general nonlocal Hamiltonian. In contrast, this Letter focuses exclusively on the class of nonlocal Hamiltonians that arise due to the use of firstprinciples nonlocal pseudopotentials for electronic struc- ture calculations.

In the pseudopotential approach, and in the absence of a magnetic field, the all-electron (AE) Hamiltonian $H^{\mathrm{AE}}=p^{2} / 2+V(\mathbf{r})$, is replaced by its pseudo (PS) equivalent, $H^{\mathrm{PS}}=p^{2} / 2+V^{\mathrm{l}}(\mathbf{r})+\sum_{\mathbf{R}} V_{\mathbf{R}}^{\mathrm{nl}} \cdot V^{\mathrm{l}}(\mathbf{r})$ is a localpotential, and $V_{\mathbf{R}}^{\text {nl }}$ is a nonlocal operator which acts only within the core region of the atomic sites, at $\mathbf{R}$ :

$$
V_{\mathbf{R}}^{\mathrm{nl}}=\int d^{3} r^{\prime} d^{3} r^{\prime \prime}\left|\mathbf{r}^{\prime}\right\rangle\left\langle\mathbf{r}^{\prime \prime}\right| V_{\mathbf{R}}^{\mathrm{nl}}\left(\mathbf{r}^{\prime}, \mathbf{r}^{\prime \prime}\right)
$$

By construction, in the valence-energy-range and to within some controllable error, (i) the eigenvalues of $H^{\mathrm{PS}}$ coincide with those of $H^{\mathrm{AE}}$, and (ii) the eigenstates of $H^{\mathrm{PS}}$ coincide with those of $H^{\mathrm{AE}}$ outside the core regions.

Turning on a magnetic field, $\mathbf{B}(\mathbf{r})=\nabla \times \mathbf{A}(\mathbf{r})$, the $\mathrm{AE}$ Hamiltonian becomes:

$$
H_{\mathbf{A}}^{\mathrm{AE}}=\frac{1}{2}\left[\mathbf{p}+\frac{1}{c} \mathbf{A}(\mathbf{r})\right]^{2}+V(\mathbf{r})
$$

The question that we aim to answer in this Letter is: what is the PS Hamiltonian that, in presence of a magnetic field, satisfies the requirements (i) and (ii) stated above? The PS Hamiltonian can be written as:

$$
H_{\mathbf{A}}^{\mathrm{PS}}=\frac{1}{2}\left[\mathbf{p}+\frac{1}{c} \mathbf{A}(\mathbf{r})\right]^{2}+V^{\mathrm{l}}(\mathbf{r})+\sum_{\mathbf{R}} V_{\mathbf{R}}^{\mathrm{nl}}+\Delta H_{\mathbf{A}},
$$

where $\Delta H_{\mathbf{A}}$ remains to be determined. $\Delta H_{\mathbf{A}}$ cannot be zero for all $\mathbf{A}(\mathbf{r})$, as $H_{\mathbf{A}}^{\mathrm{PS}}$ must be gauge invariant to satisfy (i) and (ii). Its eigenvalues must not depend on the arbitrary choice of the gauge of $\mathbf{A}(\mathbf{r})$. However, as we will demonstrate, demanding gauge invariance alone is not sufficient to uniquely determine $\Delta H_{\mathbf{A}}$.

To obtain $H_{\mathbf{A}}^{\mathrm{PS}}$ we use Blöchl's projector augmented wave (PAW) theory [3]. In the PAW approach the pseudisation procedure is defined as a linear transformation between Hilbert spaces - those of the AE valence wavefunctions, and the computationally convenient PS wavefunctions. This transformation, $|\Psi\rangle=\mathcal{T}|\tilde{\Psi}\rangle$, can be applied 
to obtain PS operators, $\tilde{\mathcal{O}}$, which correspond to their AE counterparts, $\mathcal{O}$ :

$$
\begin{aligned}
\tilde{\mathcal{O}} & =\mathcal{T}^{\dagger} \mathcal{O} \mathcal{T}=\mathcal{O}+\tilde{\mathcal{C}} \\
\tilde{\mathcal{C}} & =\sum_{i, j}\left|\tilde{p}_{i}\right\rangle\left[\left\langle\phi_{i}|\mathcal{O}| \phi_{j}\right\rangle-\left\langle\tilde{\phi}_{i}|\mathcal{O}| \tilde{\phi}_{j}\right\rangle\right]\left\langle\tilde{p}_{j}\right|,
\end{aligned}
$$

where we have adopted Blöchl's tilde to denote a PS quantity. By construction, the expectation values of $\tilde{\mathcal{O}}$ between PS wavefunctions are equal those of $\mathcal{O}$ between the corresponding AE wavefunctions. The $\left|\phi_{i}\right\rangle$ and $\left|\tilde{\phi}_{i}\right\rangle$ are atomic all electron and pseudo partial waves respectively. The projector functions $\left|\tilde{p}_{i}\right\rangle$ act only within some augmentation region - or core radius in the language of pseudopotential theory. The AE and PS partial wave coincide outside this augmentation region, and $\left\langle\tilde{p}_{i} \mid \tilde{\phi}_{i}\right\rangle=\delta_{i j}$. If the norms of the AE and PS partial waves are equal and there is just one partial wave in each angular momentum channel, which we shall assume in the following, then taking Eq. (44) with $\mathcal{O}=H^{\mathrm{AE}}$ and $\mathbf{B}(\mathbf{r})=\mathbf{0}$, we obtain the $H^{\mathrm{PS}}$ of norm-conserving pseudopotentials in the Kleinman-Bylander form [4].

In principle, in order to compute expectation values of physical observables from PS wavefunctions one should use the PS operator $\tilde{\mathcal{O}}$. In practice, since the $\mathrm{AE}$ and PS partial waves are identical outside the augmentation regions and have the same norm inside, the term $\tilde{\mathcal{C}}$ in Eq. (4) can be neglected for a large class of operators. For example, the AE perturbation Hamiltonian describing the coupling with a uniform electric field $\mathbf{E}$ is $V_{\mathbf{E}}^{\mathrm{AE}}(\mathbf{r})=\mathbf{r} \cdot \mathbf{E}$. In norm-conserving PS calculations of the electric-field response-properties, the $\tilde{\mathcal{C}}$ term is always neglected, with $V_{\mathbf{E}}^{\mathrm{AE}}(\mathbf{r})$ being used as the approximate PS perturbation potential [5, 6, 7]. The $\tilde{\mathcal{C}}$ term can also be neglected for operators for which the weight is concentrated away from the atoms or are almost constant in the augmentation regions. This is the case for operators of the form $[\mathbf{c} \cdot(\mathbf{r}-\mathbf{R})]^{n}$ in systems with a single PS atom centered at $\mathbf{R}$. Here, and in the following, $\mathbf{c}$ is a constant vector and $n \geq 0$. In addition, the $\tilde{\mathcal{C}}$ term is exactly zero for the $\mathbf{R}$-centered angular-momentum operator $\mathbf{L}_{\mathbf{R}}=(\mathbf{r}-\mathbf{R}) \times \mathbf{p}$, as the partial waves are eigenstates of $\left|\mathbf{L}_{\mathbf{R}}\right|^{2}$ and of $\left(\mathbf{L}_{\mathbf{R}}\right)_{z}$. Following this reasoning the $\tilde{\mathcal{C}}$ term is also negligible for operators of the form $\mathbf{L}_{\mathbf{R}}[\mathbf{c} \cdot(\mathbf{r}-\mathbf{R})]^{n}$. In contrast, $\tilde{\mathcal{C}}$ can not be neglected for the kinetic energy operator, $p^{2} / 2$, or for operators that are general functions of the $\mathbf{p}$ and $\mathbf{r}$ operators. Indeed, by construction, the PS wavefunctions can be expanded on a much smaller set of Fourier components in the momentum space (where $\mathbf{p}$ is diagonal) than the corresponding $\mathrm{AE}$ wavefunctions.

In general, because of the presence of the $\mathbf{p}$ operator, the $\tilde{\mathcal{C}}$ term corresponding to the AE magnetic perturbation Hamiltonian $H_{\mathbf{A}}^{\mathrm{AE}}-H^{\mathrm{AE}}$,

$$
\Delta H_{\mathbf{A}}^{\mathrm{AE}}=\frac{\mathbf{p} \cdot \mathbf{A}(\mathbf{r})+\mathbf{A}(\mathbf{r}) \cdot \mathbf{p}}{2 c}+\frac{A(\mathbf{r})^{2}}{2 c^{2}},
$$

can not be neglected, even if $\mathbf{B}(\mathbf{r})$ and $\mathbf{A}(\mathbf{r})$ are smooth on the scale of the core augmentation regions. However, as we shall show, if $\mathbf{B}(\mathbf{r})$ is smooth, and we consider a system with a single PS atom centered at $\mathbf{R}$, there is a special vector potential gauge, $\mathbf{A}^{\prime}(\mathbf{r})$, in which the $\tilde{\mathcal{C}}$ term can be neglected. This $\mathbf{A}^{\prime}(\mathbf{r})$ potential can be defined in terms of the Fourier components $\mathbf{b}_{\mathbf{G}}$ of the magnetic field,

$$
\mathbf{B}(\mathbf{r})=\sum_{\mathbf{G}}^{|\mathbf{G}|<G_{\max }} \mathbf{b}_{\mathbf{G}} e^{i \mathbf{G} \cdot \mathbf{r}},
$$

where $\mathbf{b}_{\mathbf{G}} \cdot \mathbf{G}=0$, and, if the field is smooth on the scale of the core radius $r_{\mathrm{c}}$, then $r_{\mathrm{c}} G_{\max } \ll 1$. In particular,

$$
\mathbf{A}^{\prime}(\mathbf{r})=\sum_{\mathbf{G}}^{|\mathbf{G}|<G_{\max }} \frac{1}{2} \mathbf{b}_{\mathbf{G}} \times(\mathbf{r}-\mathbf{R}) f[i \mathbf{G} \cdot(\mathbf{r}-\mathbf{R})],
$$

with $f(x)$ defined by,

$$
f(x)=2 \frac{1+x e^{x}-e^{x}}{x^{2}}=1+\frac{2}{3} x+O\left(x^{2}\right) .
$$

Note that if the field is smooth, in the core region $x \ll 1$ and $f(x)$ can be expanded in powers of $x$. Since, for a uniform magnetic field $\mathbf{A}^{\prime}(\mathbf{r})$ reduces to the symmetric gauge, $\mathbf{A}^{\prime}(\mathbf{r})$ can be seen as a generalization of the symmetric gauge to non-uniform magnetic fields.

The magnetic coupling Hamiltonian in the $\mathbf{A}^{\prime}(\mathbf{r})$ gauge is:

$$
\Delta H_{\mathbf{A}^{\prime}}^{\mathrm{AE}}=\sum_{\mathbf{G}}^{|\mathbf{G}|<G_{\max }} \frac{\mathbf{b}_{\mathbf{G}} \cdot \mathbf{L}_{\mathbf{R}} f[i \mathbf{G} \cdot(\mathbf{r}-\mathbf{R})]+c c}{4 c}+\frac{A^{\prime}(\mathbf{r})^{2}}{2 c^{2}},
$$

where $c c$ stands for the complex conjugate. All the terms in the asymptotic expansion of $\mathbf{L}_{\mathbf{R}} f[x]$ are of the form $\mathbf{L}_{\mathbf{R}}[\mathbf{c} \cdot(\mathbf{r}-\mathbf{R})]^{n}$ with $n \geq 0$. As a result, if $\mathbf{B}(\mathbf{r})$ is smooth on the scale of the augmentation region, then $\tilde{\mathcal{C}}$ term arising from the $\Delta H_{\mathbf{A}^{\prime}}^{\mathrm{AE}}$ operator can be neglected. Thus the total PS Hamiltonian in the special $\mathbf{A}^{\prime}(\mathbf{r})$ gauge is:

$$
H_{\mathbf{A}^{\prime}}^{\mathrm{PS}}=\frac{1}{2}\left[\mathbf{p}+\frac{1}{c} \mathbf{A}^{\prime}(\mathbf{r})\right]^{2}+V^{\mathrm{l}}(\mathbf{r})+V_{\mathbf{R}}^{\mathrm{nl}} .
$$

From this result we can show that the Hamiltonian for an arbitrary gauge $\mathbf{A}(\mathbf{r})$ is:

$$
\begin{aligned}
H_{\mathbf{A}}^{\mathrm{PS}}= & \frac{1}{2}\left[\mathbf{p}+\frac{1}{c} \mathbf{A}(\mathbf{r})\right]^{2}+V^{\mathrm{l}}(\mathbf{r})+\int d^{3} r^{\prime} d^{3} r^{\prime \prime}\left|\mathbf{r}^{\prime}\right\rangle\left\langle\mathbf{r}^{\prime \prime}\right| \times \\
& V_{\mathbf{R}}^{\mathrm{nl}}\left(\mathbf{r}^{\prime}, \mathbf{r}^{\prime \prime}\right) e^{\frac{i}{c} \int_{\mathbf{r}^{\prime} \rightarrow \mathbf{R} \rightarrow \mathbf{r}^{\prime \prime}} d \mathbf{r} \cdot \mathbf{A}(\mathbf{r})}
\end{aligned}
$$

where $\mathbf{r} \rightarrow \mathbf{r}^{\prime}$ indicates a straight line path from point $\mathbf{r}$ to point $\mathbf{r}^{\prime}$. To prove Eq. (12) we have just to notice that $H_{\mathbf{A}}^{\mathrm{PS}}$ is gauge invariant and reduces to Eq. (11) for $\mathbf{A}(\mathbf{r})=\mathbf{A}^{\prime}(\mathbf{r})$. 
Finally, the linearity of the Hamiltonian with respect to the electron-ion potential can be exploited to obtain $H_{\mathbf{A}}^{\mathrm{PS}}$ when there is more the one PS atom:

$$
\begin{aligned}
H_{\mathbf{A}}^{\mathrm{PS}=} & \frac{1}{2}\left[\mathbf{p}+\frac{1}{c} \mathbf{A}(\mathbf{r})\right]^{2}+V^{\mathrm{l}}(\mathbf{r})+\int d^{3} r^{\prime} d^{3} r^{\prime \prime}\left|\mathbf{r}^{\prime}\right\rangle\left\langle\mathbf{r}^{\prime \prime}\right| \times \\
& \sum_{\mathbf{R}} V_{\mathbf{R}}^{\mathrm{nl}}\left(\mathbf{r}^{\prime}, \mathbf{r}^{\prime \prime}\right) e^{\frac{i}{c} \int_{\mathbf{r}^{\prime} \rightarrow \mathbf{R} \rightarrow \mathbf{r}^{\prime \prime}} d \mathbf{r} \cdot \mathbf{A}(\mathbf{r})}
\end{aligned}
$$

We refer to $H_{\mathbf{A}}^{\mathrm{PS}}$ as the GIPAW Hamiltonian for an arbitrary magnetic field, since $H_{\mathbf{A}}^{\mathrm{PS}}$ reduces to the Hamiltonian that we have derived in our earlier work [1], if the magnetic field is uniform and if the symmetric gauge is used. Our new Hamiltonian holds if the magnetic field varies smoothly over the core region, and if the potentials are norm conserving. If the field varies more rapidly, or the norm conservation is relaxed, the $\tilde{\mathcal{C}}$ terms must be included in the Hamiltonian.

Our result differs from that of ICL. The Hamiltonian derived in Ref. 2] can be obtained from Eq. (13) if one replaces our dog-leg path $\mathbf{r}^{\prime} \rightarrow \mathbf{R} \rightarrow \mathbf{r}^{\prime \prime}$ with a straight line path $\mathbf{r}^{\prime} \rightarrow \mathbf{r}^{\prime \prime}$. The ICL Hamiltonian is also gauge invariant. In the presence of a magnetic field the value of the integral depends on the path, since $\nabla \times \mathbf{A}(\mathbf{r}) \neq$ $\mathbf{0}$. The two gauge invariant Hamiltonians are therefore different.

To clarify the situation, we examine the consequences of these differences for the calculation of the magnetic susceptibility. To compare the results obtained within the two methods with all-electron calculations, we restrict ourself to molecular systems for which it is possible to compute the susceptibility with quantum chemical approaches. The macroscopic magnetic susceptibility tensor $\overleftrightarrow{\chi}$ is defined from the second derivative of the system energy with respect to the external uniform magnetic field B:

$$
\mathbf{B} \cdot \overleftrightarrow{\chi} \cdot \mathbf{B}=-2 E^{(2)}
$$

where $E^{(2)}$ is the second order variation of the energy with respect to the magnetic field:

$$
\begin{aligned}
E^{(2)}= & 2 \sum_{o}\left[\left\langle\tilde{\Psi}_{o}^{(0)}\left|\tilde{H}^{(1)} \mathcal{G}\left(\epsilon_{o}\right) \tilde{H}^{(1)}\right| \tilde{\Psi}_{o}^{(0)}\right\rangle\right. \\
& \left.+\left\langle\tilde{\Psi}_{o}^{(0)}\left|\tilde{H}^{(2)}\right| \tilde{\Psi}_{o}^{(0)}\right\rangle\right],
\end{aligned}
$$

$\left|\tilde{\Psi}_{i}^{(0)}\right\rangle$ and $\epsilon_{i}$ are the unperturbed eigenstates and eigenvalues, $\mathcal{G}\left(\epsilon_{o}\right)=\sum_{e}\left|\tilde{\Psi}_{e}^{(0)}\right\rangle\left\langle\tilde{\Psi}_{e}^{(0)}\right| /\left(\epsilon_{o}-\epsilon_{e}\right)$ and the $o$ and $e$ sums run over occupied and empty orbitals.

In a uniform magnetic field, with the gauge $\mathbf{A}(\mathbf{r})=$ $\mathbf{B} \times \mathbf{r} / 2$, our GIPAW Hamiltonian, Eq. (13), gives rise to the following perturbation Hamiltonians:

$$
\tilde{H}_{\mathrm{GIPAW}}^{(1)}=\frac{1}{2 c}\left(\mathbf{L}+\sum_{\mathbf{R}} \mathbf{R} \times \mathbf{v}_{\mathbf{R}}^{\mathrm{nl}}\right) \cdot \mathbf{B}
$$

TABLE I: Gauge invariance test. The magnetic susceptibility of valence electrons (in $\mathrm{cm}^{3} /$ mole) of $\mathrm{CH}_{4}$ is calculated using the GIPAW and ICL approaches. $\operatorname{Tr}(\overleftrightarrow{\chi}) / 3$ is reported as a function of the distance $d$ (in a.u.) of the carbon nucleus from the gauge origin. The results are decomposed in terms of the 2 contributions present in Eq. (15).

\begin{tabular}{lrrrrrr}
$\mathrm{d}$ & $\chi_{\mathrm{GIPAW}}^{H^{(2)}}$ & $\chi_{\mathrm{GIPAW}}^{H^{(1)} \mathcal{G} H^{(1)}}$ & $\chi \mathrm{GIPAW}$ & $\chi_{\mathrm{ICL}}^{H^{(2)}}$ & $\chi_{\mathrm{ICL}}^{H^{(1)}} \mathcal{G} H^{(1)}$ & $\chi_{\mathrm{ICL}}$ \\
\hline 0.0 & -28.4 & 8.6 & -19.8 & -28.4 & 8.4 & -20.0 \\
2.5 & -68.0 & 48.2 & -19.8 & -68.0 & 48.0 & -20.0 \\
5.0 & -186.8 & 167.1 & -19.8 & -186.8 & 166.9 & -20.0 \\
7.5 & -384.9 & 365.2 & -19.8 & -384.9 & 364.9 & -20.0 \\
10.0 & -662.2 & 642.5 & -19.8 & -662.2 & 642.3 & -20.0
\end{tabular}

and

$$
\tilde{H}_{\mathrm{GIPAW}}^{(2)}=\frac{1}{8 c^{2}}\left[(\mathbf{B} \times \mathbf{r})^{2}+\sum_{\mathbf{R}} \mathbf{B} \times \mathbf{R} \cdot \stackrel{\mathrm{D}}{\mathbf{R}}_{\mathbf{n}} \cdot \mathbf{B} \times \mathbf{R}\right],
$$

where $\mathbf{v}_{\mathbf{R}}^{\mathrm{nl}}=\left[\mathbf{r}, V_{\mathbf{R}}^{\mathrm{nl}}\right] / i, D_{\mathbf{R}, \alpha, \beta}^{\mathrm{nl}}=-\left[r_{\alpha},\left[r_{\beta}, V_{\mathbf{R}}^{\mathrm{nl}}\right]\right]$, and $\alpha$ and $\beta$ are Cartesian indexes.

The corresponding perturbation Hamiltonians obtained following the ICL approach are

$$
\tilde{H}_{\mathrm{ICL}}^{(1)}=\frac{1}{2 c}(\mathbf{r} \times \mathbf{v}) \cdot \mathbf{B},
$$

$$
\tilde{H}_{\mathrm{ICL}}^{(2)}=\frac{1}{8 c^{2}}\left[(\mathbf{B} \times \mathbf{r})^{2}+\sum_{\mathbf{R}} \mathbf{B} \times \mathbf{r} \cdot \overleftrightarrow{\mathbf{D}}_{\mathbf{R}}^{\mathrm{nl}} \cdot \mathbf{B} \times \mathbf{r}\right],
$$

where $\mathbf{v}=\mathbf{p}+\sum_{\mathbf{R}} \mathbf{v}_{\mathbf{R}}^{\mathrm{nl}}$.

We compute $\overleftrightarrow{\chi}$ in molecules with both the GIPAW and the ICL approaches. We describe the electronic structure with density functional theory in the local density approximation. We use a large-cubic-periodic supercell of $6000 \mathrm{au}^{3}$, in order to avoid the interaction of the molecules with their periodic replica, and TroullierMartins pseudopotentials [8] in the Kleinman-Bylander form [4]. We expand the wavefunctions in plane-waves with a cutoff of 100 Ry. The position operator is not defined within periodic boundary conditions. We treat it approximately by constructing a periodic saw-tooth like function centered on the molecules. For large cells this operator well approximates the position operator where the electron density is not negligible [1]. The contribution of core electrons to magnetic properties can not be neglected. This contribution is however rigid, i.e. independent of the chemical environment [1, 9]. We compute the core contribution with an atomic code.

Both the GIPAW and the ICL approaches are, by construction, gauge invariant. To verify that our numerical implementation and the use of a finite basis set preserve this property, we compute $\chi$ for a $\mathrm{CH}_{4}$ molecule as a function of the distance between the gauge origin and the molecular center. The results summarized in Table show that the calculated $\chi$ is indeed gauge invariant, 
TABLE II: The principal values of the magnetic susceptibility tensor (in $\mathrm{cm}^{3} /$ mole) calculated for a selection of small molecules. The IGAIM results are obtained from the Gaussian98 [10] code using the aug-cc-pVQZ basis set for $\mathrm{H}$ and the aug-cc-pCVQZ basis sets for the remaining elements 11]. We include in the GIPAW and ICL results the rigid core contributions obtained using an atomic code. The GIPAW and ICL results are indicated as deviations from the IGAIM results. The root mean square (RMS) and maximum absolute deviations and percentage deviations for the two methods are reported at the foot of the table.

\begin{tabular}{|c|c|c|c|}
\hline Mol. & IGAIM & $\Delta$ GIPAW & $\triangle \mathrm{ICL}$ \\
\hline $\mathrm{C}_{6} \mathrm{H}_{6}$ & $.26-33.22-33$. & $-.77-.15-.15$ & $\begin{array}{lll}-2.4 & -5.3 & -5.3\end{array}$ \\
\hline $\mathrm{CF}_{4}$ & $-33.01-33$ & $-.45-.45-.45$ & $\begin{array}{lll}-1.7 & -1.7 & -1.7\end{array}$ \\
\hline $\mathrm{CH}_{3} \mathrm{~F}$ & $2-16.00-16$ & $-.14-$ & $-0.4-0.6$ \\
\hline $\mathrm{CNH}_{5}$ & $9-2$ & $-.12-$ & -0.4 \\
\hline $\mathrm{CH}_{4}$ & $-19.87-1$ & $-.06-.06$ & $\begin{array}{lll}-0.3 & -0.3 & -0.3\end{array}$ \\
\hline $\mathrm{CO}$ & $\begin{array}{lll}-18.35 & -9.63 & -9.63\end{array}$ & $-.02-.14-$ & $\begin{array}{lll}-0.1 & -2.2 & -2.2\end{array}$ \\
\hline $\mathrm{HCP}$ & $-35.23-25.62-25.62$ & $-.01-.09-.09$ & $0.4-2.5-2.5$ \\
\hline $\mathrm{P}_{2}$ & $-46.13-18.23-18$. & $\begin{array}{lll}-.01 & .03 & .23\end{array}$ & $\begin{array}{lll}0.6 & -5.9 & -5.9\end{array}$ \\
\hline $\mathrm{PF}_{3}$ & $\begin{array}{llll}-28.08 & -27.17 & -27.11\end{array}$ & $\begin{array}{lll}-.40 & -.29 & -.29\end{array}$ & $\begin{array}{lll}-2.7 & -2.9 & -2.9\end{array}$ \\
\hline $\mathrm{Si}_{2} \mathrm{H}_{4}$ & $-44.14-39.24-37.06$ & $\begin{array}{lll}-.10 & -.09 & -.22\end{array}$ & $-1.5-1.6-2.9$ \\
\hline $\mathrm{SiF}_{4}$ & $\begin{array}{lll}-40.92 & -40.92 & -40.92\end{array}$ & $-.34-.34-.34$ & $\begin{array}{lll}-1.6 & -1.6 & -1.6\end{array}$ \\
\hline $\mathrm{SiH}_{3} \mathrm{~F}$ & $-27.77-18.63-18.63$ & $-.13-.17-.17$ & $\begin{array}{lll}-0.9 & -1.2 & -1.2\end{array}$ \\
\hline $\mathrm{SiH}_{4}$ & $\begin{array}{lll}-23.97 & -23.97 & -23.97\end{array}$ & $-.08-.08-.08$ & $\begin{array}{lll}-0.7 & -0.7 & -0.7\end{array}$ \\
\hline
\end{tabular}

$\begin{array}{lcc}\operatorname{RMS} \Delta & 0.25 & 2.35 \\ \operatorname{Max}|\Delta| & 0.77 & 5.85 \\ \operatorname{RMS} \% & 0.9 & 11.0 \\ \operatorname{Max}|\%| & 1.9 & 32.1\end{array}$

while the individual terms due to $\tilde{H}^{(1)}$ and $\tilde{H}^{(2)}$ clearly are not.

In order to obtain an independent assessment of the accuracy of the two methods, we compare to a truly all electron method, the individual gauges for atoms in molecules (IGAIM) method 12] as implemented in Gaus$\operatorname{sian} 98$ [10]. The magnetic susceptibility converges only slowly with Gaussian or atomic basis sets. The aug-cc$\mathrm{p}(\mathrm{C}) \mathrm{VxZ}$ basis set series [11] has been previously shown exhibit reliable convergence for magnetic response properties [9, 13, and we confirm this by converging the magnetic susceptibility for $\mathrm{CH}_{4}$ by using up to the aug-ccpCV5Z basis for C (and aug-cc-pV5Z for $\mathrm{H}$ ). For the remaining calculations we use the corresponding quadruple zeta basis sets, at which level the $\mathrm{CH}_{4}$ result is converged to better than $0.1 \mathrm{~cm}^{3} /$ mole. Indeed, calculations using the largest basis sets rapidly become intractable for even moderately sized molecules. The results are summarized in Table III and show that the GIPAW method results in values for the magnetic susceptibility that are consistently closer to the all electron results than those calculated using the ICL method by roughly an order of magnitude. E.g., the ICL results for $\mathrm{P}_{2}$ deviate by almost one third of the total, while the maximum fractional deviation for our method is less than two percent.

We have derived, and demonstrated the practical utility of, a theory for the coupling of nonlocal pseudopotentials to arbitrary electromagnetic fields. While the ICL method may be the best possible for an arbitrary nonlocal Hamiltonian we have shown that by focusing on the nonlocal Hamiltonian most frequently encountered in electronic structure calculations we are able to make considerable improvements. This is of great importance if the results are to be compared quantitatively with experiment.

The work of CJP was supported by the Universités Paris 6 and 7 in France, and the EPSRC in the United Kingdom. We thank the High Performance Computing Facility of the University of Cambridge for access to Hodgkin, their SGI Origin 2000, and the CNRS funded IDRIS supercomputing center. We would also like to thank Thomas Gregor for the molecular geometries, and Kirk Peterson for his kind help with the Gaussian basis functions.

[1] C. J. Pickard and F. Mauri, Phys. Rev. B 63, 245101 (2001).

[2] S. Ismail-Beigi, E. K. Chang, and S. G. Louie, Phys. Rev. Lett. 87, 087402 (2001).

[3] P. E. Blöchl, Phys. Rev. B 50, 17953 (1994).

[4] L. Kleinman and D. Bylander, Phys. Rev. Lett. 48, 1425 (1982).

[5] S. Baroni and R. Resta, Phys. Rev. B 33, 7017 (1986).

[6] S. de Gironcoli, S. Baroni, and R. Resta, Phys. Rev. Lett. 62, 2853 (1989).

[7] R. D. King-Smith and D. Vanderbilt, Phys. Rev. B 47, 1651 (1993).

[8] N. Troullier and J. L. Martins, Phys. Rev. B 43, 1993 (1991).

[9] T. Gregor, F. Mauri, and R. Car, J. Chem. Phys. 111, 1815 (1999).

[10] M. J. Frisch, G. W. Trucks, H. B. Schlegel, G. E. Scuseria, M. A. Robb, J. R. Cheeseman, V. G. Zakrzewski, J. A. Montgomery, Jr., R. E. Stratmann, et al., Gaussian 98 (Revision A.11), Gaussian, Inc., Pittsburgh PA (2001).

[11] K. A. Peterson and J. T. H. Dunning, J. Chem. Phys. 117, 10548 (2002).

[12] T. A. Keith and R. F. W. Bader, Chem. Phys. Letters 194, 1 (1992).

[13] T. Helgaker, M. Jaszuński, and K. Ruud, Chemical Reviews 99, 293 (1999). 